\title{
Controle de acesso para condomínios residenciais
}

\author{
Bruno Serra $^{1}$, Danilo Evangelista ${ }^{2}$, Fábio Lima ${ }^{1}$ \\ ${ }^{1}$ Instituto Federal do Piauí (IFPI) \\ ${ }^{2}$ Centro Universitário Facid Wyden - (UniFacid Wyden) \\ brunoserrabregmail.com, danilo.evangelista@facid.edu.br,fabio@ifpi.edu.br
}

\begin{abstract}
Access control is an essential feature when developing a service aimed at residential condominiums. However, for this service to perform as expected it is necessary to identify the whole process and thus choose the most appropriate techniques. The security aspect, in this sense, is the most desired aspect for the service to be reliable. This paper presents an access control mechanism used to ensure reliable access control (CA) for residents and their guests. The CA developed uses the concept of screening in order to ensure the principles of identification, authentication and authorization, allowing access to those guests who are really legitimate. A preliminary assessment of the CA service was conducted to assess the degree of satisfaction of residents and employees of a residential condominium.
\end{abstract}

Resumo. O controle de acesso é uma funcionalidade essencial quando se desenvolve um serviço voltado para condomínios residenciais. Contudo, para que esse serviço performe de acordo com o esperado é preciso identificar o processo como todo e assim escolher as técnicas mais adequadas. $O$ aspecto segurança, nesse sentido, é quesito mais desejado para que o serviço seja confiável. Este trabalho apresenta um mecanismo de controle de acesso utilizado cujo o objetivo é garantir o controle de acesso (CA) confiável para os moradores e seus respectivos convidados. $O C A$ desenvolvido utiliza o conceito de triagem com o intuito de garantir os princípios de identificação, autenticação e autorização, liberando o acesso aqueles convidados que realmente são legítimos. Uma avaliação preliminar do serviço de CA foi conduzida para avaliar o grau de satisfação de moradores e colaboradores de um condomínio residencial.

\section{Introdução}

Atualmente, com a transição da sociedade da "disciplina"para sociedade do "controle", onde a preocupação com a ordem social tem o foco no grupo e não mais no indivíduo, os condomínios residenciais surgem como novos espaços urbanos em expansão no Brasil [BATISTA 2015]. A eficiência nos gastos, a melhor qualidade de vida e um maior senso de segurança são fatores intrínsecos ao fenômeno que impulsionam essa transformação. Com a nova tendência, nasce também novas oportunidades para aplicação de tecnologias de gerenciamento e controle inteligentes na habitual área de automação residencial e condominial [de Oliveira Schwartz 2018].

O uso da tecnologia é fundamental para diminuir custos e otimizar processos administrativos dos condomínios residenciais [de Oliveira Schwartz 2018]. Existem soluções que variam desde monitoramento e controle eficiente do consumo de água até gerenciamento de funções administrativas como, reserva de áreas comuns, transparência no 
pagamento de contas, facilitação dos processos democráticos em reuniões prediais, etc. [de Oliveira Schwartz 2018, Bento 2016, Nunes 2019].

Apesar das diversas abordagens propostas para automação condominial, pouco tem sido desenvolvido na direção do gerenciamento inteligente do controle de acesso de pessoas ao condomínio. Quais os stakeholders envolvidos nesse processo? Quais os requisitos importantes a serem considerados no projeto de um mecanismo de controle de acesso? Este trabalho investiga como gerenciar o acesso a um condomínio residencial de forma segura, eficiente e adaptativa a diferentes situações e contextos[Carvalho 2015].

Este trabalho apresenta um estudo de caso onde o processo de controle de acesso ao um condomínio residencial foi analisado e modelado em um mecanismo tecnológico para gerenciamento inteligente deste processo. O mecanismo de controle de acesso proposto, emprega um modelo de triagem no qual leva em consideração três princípios, identificação, autenticação e autorização. Esta solução também explora as tecnologias o stack React Native e JS, bem como do framework Django implementado em Python

O restante deste trabalho está organizado da seguinte maneira: A Seção 2 apresenta os trabalhos relacionados e discute suas deficiências. A Seção 3 compreende o desenvolvimento do mecanismo de controle de acesso e a metodologia empregada no dedsenvolvimento.Na Seção 4 uma análise preliminar da solução é apresentada. Por fim, a Seção 4.1 conclui o trabalho e discute os trabalhos futuros.

\section{Trabalhos Relacionados}

Controle de acesso (CA) é uma prática desenvolvida não só no âmbito computacional, e assim a literatura já aborda esse tema de uma maneira vasta e ampla. Atualmente, muitas aplicações web e mobile implementam esse serviço para liberar acesso conforme a permissão de cada usuário diante do recurso [Khezr et al. 2019]. Tendo em vista esse cenário, a automatização dos processos de um CA tem como consequência a alteração dos requisitos. Comparando-se um CA manual, onde se tenha eventos de alta demanda, com o CA automatizado, este último terá resultados satisfatórios em relação a qualidade de serviço (QoS), uma vez que apresenta uma performance superior devido a velocidade de identificação, autenticação e autorização realizadas neste processo.

Os três aplicativos mais utilizado pelos condomínios no cenário nacional serão analisados através dos requisitos mencionados anteriormente. Esses três aplicativos são uCondo, Severino e APPTOHOME, ambos estão disponíveis para as plataformas Android e iOS, e apresentam a funcionalidade CA automatizado para condomínios residenciais. Além da avaliação baseada nos requisitos, os pontos positivos e negativos serão levantados como uma forma de validação dos requisitos para a escolha das técnicas mais adequadas para um aplicativo deste gênero.

O aplicativo uCondo [Ucondo 2018] consiste numa plataforma orientada à serviços condominiais, no qual o CA é uma das funcionalidades contempladas por esse app. Dentre elas estão controle de reservas, documentos, enquetes, classificados, financeiro e CA. Essa última funcionalidade inicia através do cadastro realizado pelo residente de seus respectivos convidados. Para agendar uma data de visita, basta o residente marcar uma data desejada que o sistema fará a reserva. Em seguida, um QR Code será gerado para que o convidado apresente quando chegar ao condomínio. O convidado deve passar pelo processo de triagem, no qual será submetido pelas fases de identificação, autenticação e 
autorização. Por fim, esse convidado terá acesso ao condomínio e o residente será notificado por uma mensagem via aplicativo.

O processo de triagem realizado pelo uCondo também acontece nos aplicativos Severino [SeverinoAPP 2017] e APPTOHOME [Apptohome 2017]. Tanto o Severino quanto APPTOHOME são soluções que auxiliam a gestão condominial através das suas respectivas funcionalidades. O aplicativo Severino possui as seguintes funcionalidades: quadro de avisos, controle financeiro, reservas de áreas em comum, gestão de visitantes, correios, documentos e enquetes. Da mesma forma o APPTOHOME implementa as mesmas funcionalidades, além disso este aplicativo ainda possui uma versão white label onde um condomínio pode ter o seu app totalmente personalizado com o seu respectivo nome e identidade visual. Contudo, os três aplicativos mencionados empregam $Q R C O D E$ que possui algumas vulnerabilidades relativas à confidencialidade.

Para implementar um CA para condomínios residenciais este trabalho considera os seguintes requisitos, identificação, autenticação, autorização e confiabilidade. Os três requisitos iniciais estão relacionados ao processo de triagem, onde, um convidado recebe o seu token gerado por um morador mediante a confirmação da sua visita. Assim, um token será associado a sua identidade para que ele apresente este código no acesso ao condomínio. Quando esse convidado chegar ao residencial basta apresentar o token para que ele seja identificado, e que inicie o processo de triagem cujo objetivo é certificar a identidade e o seu respectivo acesso ao condomínio.

\section{Controle de acesso confiável para condomínios residenciais}

O mecanismo de controle de acesso implementado neste trabalho faz parte de uma plataforma de gestão de condomínios residenciais. Essa plataforma tem como objetivo automatizar todo o processo de administração de um condomínio, isto é, gestão dos documentos, reservas de áreas em comuns, avisos, e o controle de acesso (CA). Para realizar o CA, o mecanismo emprega um modelo de triagem no qual leva em consideração três princípios, identificação, autenticação e autorização. O stack de tecnologias utilizadas para o desenvolvimento mobile e web são o React Native e JS, o framework Django, escrito em Python. A infraestrutura de cloud escolhida para suportar a plataforma foi a arquitetura servless implementada pela AWS (Amazon Web Services).

A plataforma consiste a partir de um sistema distribuído composto por dois módulos, o backend e o frontend. O primeiro módulo, realiza a gestão dos usuários do condomínio, os moradores, os convidados, e os colaboradores. Essa parte do sistema também lida com as requisições dos usuários, via aplicativo, que são manipuladas através de uma API (Aplication Progaming Interface), inicialmente, e posteriormente, quando necessário elas são tratadas no backend. Enquanto a segunda parte, tem como objetivo suprir a demanda dos moradores por meio de interfaces interativas.

O fluxo de dados realizado pela plataforma está representado na Figura 1(a). Dessa maneira, quando um usuário realizar o acesso de seu dispositivo móvel a API consumirá a requisição feita, como representado no passo 1. Caso seja o seu primeiro acesso, a API redireciona esta requisição para o agente autenticador de cadastro, passo 2. Este passo é importante para confirmar a legitimidade do usuário na plataforma sendo executado apenas uma vez. Assim que for aprovado, a API pode trocar dados com backend, quando preciso, e retornar as informações necessárias para a aplicação mobile, como ilus- 
trado no fluxo 1-3. O agendamento de visita pode ser um exemplo de interação com o backend, onde a API registra as informações no banco de dados e processa o token de acesso para o visitante como resposta.

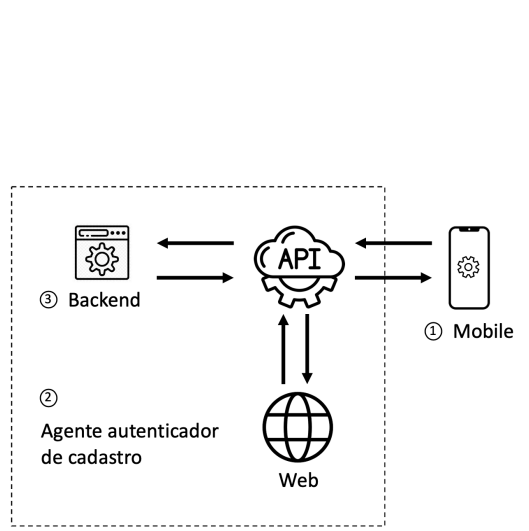

(a) Arquitetura da Plataforma

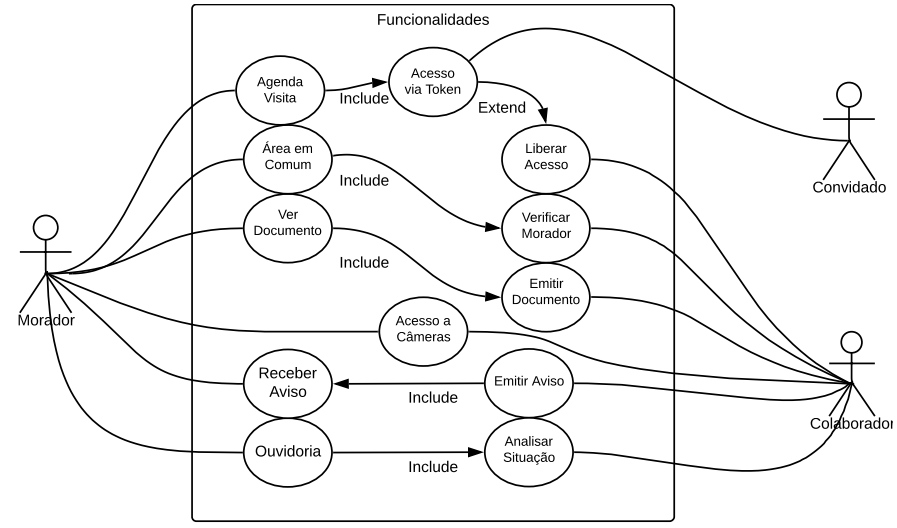

(b) Funcionalidades da Plataforma e seus atores

Figura 1. Visão Geral da Plataforma

A Figura 1(b) apresenta o diagrama de caso de uso de funcionalidades e os respectivos atores, isto é, moradores, convidados e colaboradores. Os moradores são o público alvo da plataforma, onde eles interagem com ela através do aplicativo. Um morador pode acessar documentos, agendar visita, acessar câmeras, receber avisos, e solicitar o acesso de visitantes. Já um convidado tem seus dados registrados na plataforma por motivos de controle e para facilitar a identificação em caso de alguma suspeita. A sua interação com a plataforma consiste na utilização do token gerado por um morador através da sua lista de convidados para que ele tenha acesso ao condomínio. O colaborador interage com a plataforma cadastrando usuários (moradores e novos colaboradores), emitindo documentos de assembleias, acessando as câmeras do condomínio, criando avisos, autenticando e autorizando acesso de convidados. Assim, cada ator interage com uma parte do sistema para que o gerenciamento, principalmente do controle de acesso aconteça de forma confiável.

\subsection{Controle de Acesso para condomínios}

O controle de acesso (CA) desenvolvido neste trabalho será apresentado desde a sua representação matemática até o passo a passo feito no aplicativo. A representação matemática, Figura 2(a), visa inicialmente levantar as entidades e assim como o seu relacionamento através de funções e teoria de conjuntos. Esta abordagem é abstrata porém, ela auxilia no entendimento do comportamento do CA sem necessitar de uma implementação prévia. Em seguida, a Figura 2(b) representa uma modelagem baseada em diagramas de caso de uso que por sua vez tem como objetivo a especificação de processos pertinentes ao CA. Por fim, a o funcionamento prático do CA dentro da plataforma no qual está ilustrado nas Figuras 3(a) até a 4(b).

$\mathrm{O}$ processo de modelagem analítica considera um conjunto universo CA e três conjuntos, Moradores $(\mathbf{M})$, Colaboradores $(\mathbf{C})$ e Visitantes $(\mathbf{V})$. O controle de acesso tem como principal objetivo facilitar o fluxo de visitantes de um codomínio, onde por intermédio de um colaborador um token gerado pelo morador deve ser apresentado por este convidado. Considerando a modelagem proposta, para cada conjunto pertencente a CA uma função bijetora deverá ser atribuída para o seu relacionamento.A propriedade 


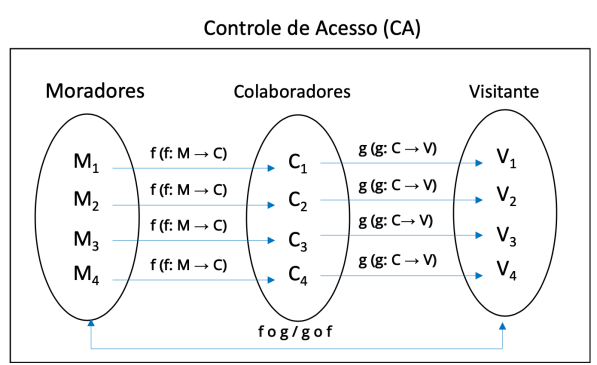

(a) Modelagem para o controle de acesso

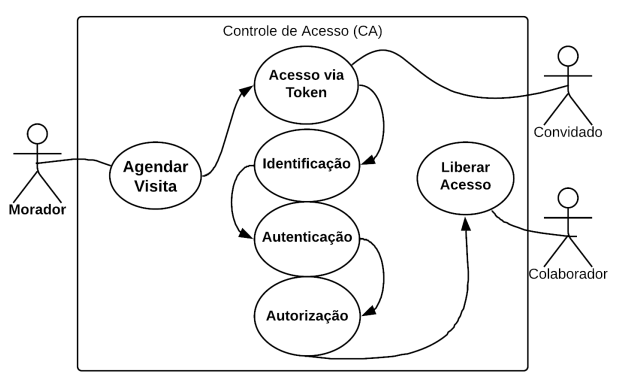

(b) Diagrama de Classes para o Controle de Acesso

Figura 2. Modelagem e Diagrama de Classes - CA da plataforma

Bijetiva possibilita em uma dada função $\mathrm{f}: \mathrm{A} \rightarrow \mathrm{B}$ que os elementos de A possua como imagem elementos distintos em B. Neste tipo de relacionamento não existe dois elementos distintos de A com a mesma imagem em B. O relacionamento inverso também é possível, ou seja, $\mathrm{f}: \mathrm{B} \rightarrow \mathrm{A}$ obtida por meio da função inversa. Logo, esta propriedade garante que o fluxo entre um morador, colaborador e visitante seja único, ou seja, nenhuma das entidades podem se relacionar com mais de um elemento de outro conjunto pertencente ao CA.

As entidades possuem os seus relacionamentos modelados através de seis funções, $f, f^{-1} g, g^{-1}, f o g, g o f$. Inicialmente, um Morador $\mathbf{M}$ deve solicitar o agendamento ao Colaborador $\mathbf{C}$ por meio da função $\mathrm{f}: \mathrm{M} \rightarrow \mathrm{C}$ e obter retorno por meio de $f^{-1}$ : $\mathrm{C}$ $\rightarrow \mathrm{M}$. De forma análoga, g: $\mathrm{C} \rightarrow \mathrm{V}$ e $f^{-1}: \mathrm{V} \rightarrow \mathrm{C}$ representam a interação entre os conjuntos $\mathbf{C}$ e V. Por fim, os conjuntos $\mathbf{M}$ e $\mathbf{V}$ utilizam das funções compostas, $f o g$, para o sentido Morador-Visitante e $g o f$, uma vez que o fluxo for Visitante-Morador.

O CA está ilustrado no diagrama de caso de uso da Figura 2(b). Neste diagrama é possível observar o processo como todo desde o agendamento de uma visita até a autorização para que um convidado adentre ao condomínio. Um morador que desejar realizar algum evento deve informar para o sistema o dia que seu convidado virá. Em seguida, um código token é gerado para que possa ser compartilhado ao convidado. Ao chegar no condomínio, o convidado passará pela triagem de autenticação. Feito este processo, ele terá o acesso autorizado ou negado de acordo com o resultado da triagem.

O primeiro caso de uso que será discutido diz respeito a interação entre um morador e o agendamento de uma visita. A Figura 3(a) mostra a tela inicial do módulo de CA, onde um morador deve cadastrar um novo convidado tocando no símbolo "+"localizado no topo superior direito. Este cadastro,Figura 3(b), é bem simples uma vez que ele consiste da foto, nome, RG. O aplicativo provê uma lista de favoritos e caso esta opção seja marcada ele será destacado na lista de visitantes como mostra a Figura . Para agendar uma visita, basta que o morador selecione o nome de um visitante cadastrado e escolha a opção agendar visita. Por fim, ele será direcionado para o agendamento,Figura 3(d), onde o morador precisa escolher a data de visita desejada.

A Figura 4(a) mostra como um morador gera o token para que ele seja compartilhado com o seu respectivo convidado. As formas de compartilhamentos possíveis do token são email, mensagem, e whatsapp. Quando um morador gera o código token, automaticamente o código é compartilhado com o colaborador responsável pelo processo de autenticação que está portaria do condomínio. Ele que mantém a lista dos visitantes do 


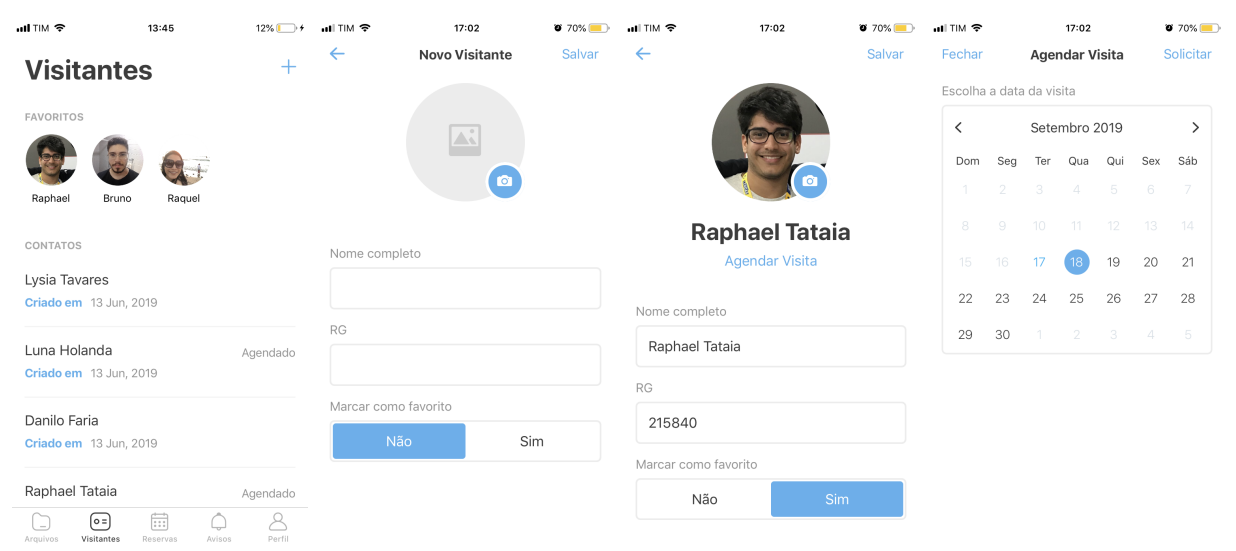

(a) Listagem de Visitantes (b) Cadastro de Visitante (c) Detalhamento do Visi- (d) Agendamento de visi-

Figura 3. Interação morador agendamento de visita

condomínio. Esta lista tem uma visão diária, semanal, e mensal, possibilitando o colaborador identificar dias de alta demanda. O condomínio então pode utilizar esses dados para entender o padrão de visitas por residência com o objetivo de otimizar o processo de gestão do condomínio.

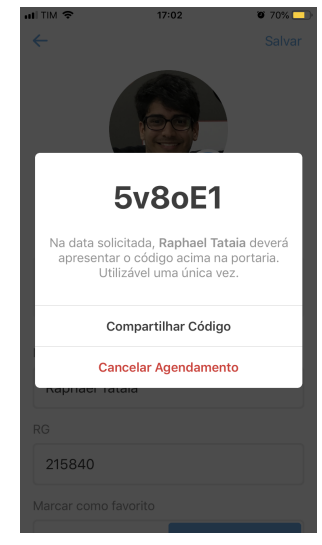

(a) Token de visita

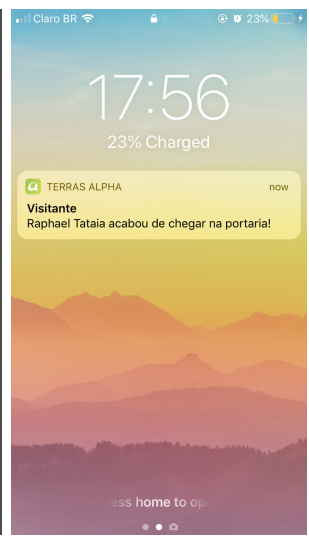

(b) Notificação para o morador

Figura 4. Interação morador agendamento de visita

O convidado ao chegar no condomínio apresenta o token compartilhado pelo morador. Em seguida inicia-se o processo de triagem, no qual está representado Figura 2(b). Este processo tem início com a identificação do convidado através do seu nome e do código token associado ao seus perfil. O colaborador checa os dados passados autenticando o convidado por meio do sistema web junto à portaria. Caso o código token seja validado, o processo de triagem encerra com a sua autorização e consequentemente o acesso ao condomínio. Por fim, o morador recebe uma notificação avisando que o convidado está à caminho da sua residência, representada pela Figura 4(b).

\section{Avaliação preliminar}

Uma vez que o serviço de gestão automatizada é implantado num condomínio o fator satisfação do cliente, neste caso moradores e colaboradores, tem um peso importante. A plataforma e o serviço de controle de acesso foram avaliados através aspec- 
tos de qualidade de serviço (QoS). Para avaliar o grau de satisfação desses stakeholders uma pesquisa advinda de um formulário que emprega a metodologia NPS (Net Promote Score) [Owen 2019]. Essa pesquisa considerou um espaço amostral de 100 moradores, 20 colaboradores, totalizando 120 pessoas. Com espaço o amostral considerado é possível obter uma um erro médio de 5\%, obtendo assim um grau de confiança de $95 \%$.

O NPS é uma metodologia simples cujo o objetivo é definir o nível de fidelidade dos cliente em detrimento a um serviço. O seu o objetivo então é calcular a satisfação do cliente, e com isso tomar decisões de melhorias contínuas, caso exista algum processo abaixo do esperado deve-se destinar uma contingência. O cálculo do NPS acontece inicialmente com um pesquisa os participante dela atribuem notas aos questionamentos. De forma individual, cada participante recebe uma classificação através da sua nota dada ao serviço. Assim, eles podem ser promotores, detratores ou neutros, conforme a sua respectiva nota.

$$
N P S=\frac{\sum(\text { Promotores }- \text { Detratores })}{\text { TotaldeRespostas }} X 100
$$

Tabela 1. Questionário para aferir NPS

\begin{tabular}{|c|l|}
\hline Número & \multicolumn{1}{c|}{ Questionamento } \\
\hline 1 & Como foi o desempenho serviço (Plataforma) ? \\
\hline 2 & O serviço atendeu suas expectativas? \\
\hline 3 & Qual o nível de confiança serviço CA? \\
\hline 4 & Pretende usar a plataforma novamente? \\
\hline 5 & Você recomendaria esse serviço? \\
\hline
\end{tabular}

A partir dessa métrica, um conjunto de respostas associadas à serviços julga a sua qualidade por meio de notas que variam numa escala de 0 à 10 . Os Promotores são clientes que atribuíram notas 9 e 10. Estes clientes realmente ficaram satisfeitos com serviço. Já os Neutros são aqueles que deram notas entre 7 e 8. Por fim, os Delatores são os que consideraram notas abaixo de 6.Desta forma, para obter o NPS devemos aplicar o seguite calculo, (Eq.1).

O desenvolvimento do formulário é uma parte importante do processo da avaliação de satisfação feita através do NPS. O questionário considerado por esta trabalho conta com cinco questões que são simples e diretas. Todos os 150 participantes possuem no mínimo 45 dias de uso na plataforma, e o período de avaliação durou 1 semana através de um formulário digital. Os cinco questionamentos estão descritos na Tabela 1. Eles são os mais utilizados quando uma avaliação de satisfação é conduzida.

\subsection{Resultados}

O Gráfico 5(a) apresenta os resultados coletados através da pesquisa de satisfação, onde o critério escolhido é o NPS. Nessa avaliação o resultado desse quesito variou entre $70 \%$ à $85 \%$ considerando um intervalo de confiança de $5 \%$. Tendo em vista esses resultados fica evidente que a plataforma performou bem no que diz respeito a qualidade de satisfação. Observando como referência a tabela de classificação do NPS [Owen 2019] é possível concluir que para os resultados acima de $75 \%$, o serviço é considerado como de excelência.

Uma outra perspectiva avaliada por este trabalho consiste em analisar as notas atribuídas ao serviço. Esse resultado está contido no Gráfico 5(b). No gráfico é possível 


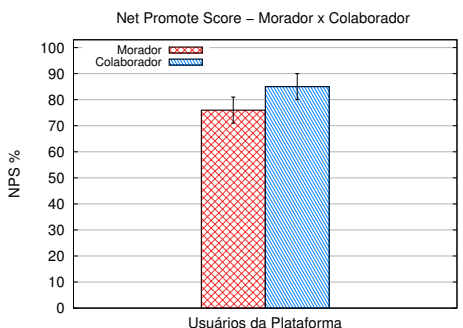

(a) NPS para moradores e colaboradores

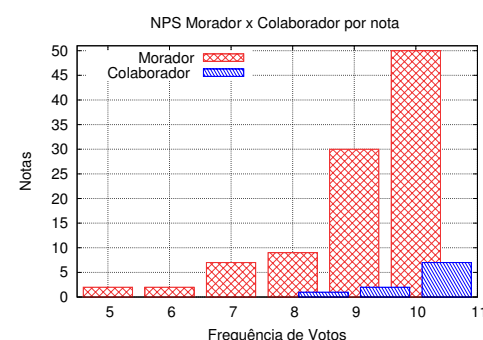

(b) Notas atribuidas pelos moradores e colaboradores

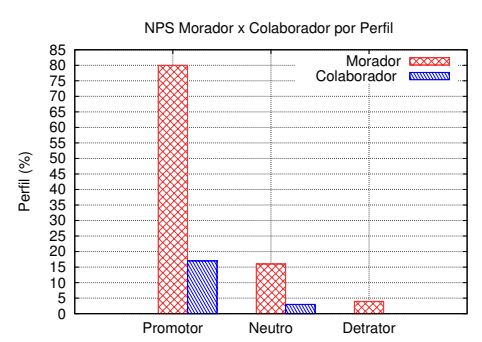

(c) NPS para moradores e colaboradores

Figura 5. Avaliação da plataforma atavés do NPS

observar que a quantidade de avaliações entre as notas 9 e 10 fazem parte da moda amostral em ambos os cenários, moradores e colaboradores. Com esta informação é possível afirmar que tanto os colaboradores quanto os moradores confiam no serviço da plataforma, uma vez que ela atende às suas necessidades. Paralelamente a esta constatação, o Gráfico 5(c) complementa essa conclusão. Isto ocorre pois esse gráfico caracteriza o perfil dos usuários da plataforma. Deste modo, temos como promotores aqueles que estão satisfeitos e confiam no serviço prestado.

\section{Conclusão}

Este trabalho apresentou um mecanismo para o controle de acesso confiável em condomínios residenciais. Este mecanismo leva em consideração o processo de triagem e a utilização de tokens para suportar o controle de acesso realizado plataforma. A avaliação do mecanismo foi conduzida por meio de formulários de satisfação utilizando métrica Net Promote Score (NPS). Os resultados demonstram através de uma avaliação prévia que o mecanismo assim como a plataforma são confiáveis, alcançando um patamar de excelência de serviço. Como trabalhos futuros pretende-se automatizar o processo de triagem, considerar prestadores de serviços no controle de acesso, assim como avaliar a performance da plataforma e do controle de acesso em condomínios residenciais de maiores instâncias.

\section{Referências}

Apptohome (2017). https://apptohome.com.br/.

BATISTA, R. L. (2015). Produção do espaço urbano e controle social: os espaços residenciais populares fechados como novo modelo de moradia. PhD thesis, Tese de Doutorado. Programa de Pós Graduação em Geografia. FCT-UNESP.

Bento, B. R. (2016). Automação aplicada à redução do desperdício de água em instalações prediais.

Carvalho, J. C. D. R. S. (2015). A atuação do gestor de segurança em condomínios residenciais. Segurança Privada-Unisul Virtual.

de Oliveira Schwartz, R. B. (2018). Revolucionando o condomínio. Editora Saraiva.

Khezr, S., Moniruzzaman, M., Yassine, A., and Benlamri, R. (2019). Blockchain technology in healthcare: A comprehensive review and directions for future research. Applied Sciences, 9(9):1736.

Nunes, L. d. S. (2019). Siscond: um sistema para gestão de condomínios.

Owen, R. (2019). Net promoter score and its successful application. In Marketing Wisdom, pages 17-29. Springer.

SeverinoAPP (2017). https://severinoapp.com/.

Ucondo (2018). https://ucondo.com.br/. 